\title{
Cylindrically Symmetric, Asymptotically Flat, Petrov Type D Spacetime with a Naked Curvature Singularity and Matter Collapse
}

\author{
Faizuddin Ahmed \\ Department of Physics, Cotton College, Guwahati 781 001, India \\ Correspondence should be addressed to Faizuddin Ahmed; faizuddinahmed15@gmail.com
}

Received 1 December 2016; Revised 17 January 2017; Accepted 1 February 2017; Published 6 March 2017

Academic Editor: Elias C. Vagenas

Copyright (C) 2017 Faizuddin Ahmed. This is an open access article distributed under the Creative Commons Attribution License, which permits unrestricted use, distribution, and reproduction in any medium, provided the original work is properly cited. The publication of this article was funded by $\mathrm{SCOAP}^{3}$.

\begin{abstract}
We present a cylindrically symmetric, Petrov type D, nonexpanding, shear-free, and vorticity-free solution of Einstein's field equations. The spacetime is asymptotically flat radially and regular everywhere except on the symmetry axis where it possesses a naked curvature singularity. The energy-momentum tensor of the spacetime is that for an anisotropic fluid which satisfies the different energy conditions. This spacetime is used to generate a rotating spacetime which admits closed timelike curves and may represent a Cosmic Time Machine.
\end{abstract}

\section{Introduction}

A spacetime is said to have singularities if it possesses inextensible curves with finite affine length. The singularities of spherically symmetric matter filled spacetimes can be recognized from the divergence of the energy density and curvature scalars, for example, the Kretschmann scalar $R_{\mu \nu \rho \sigma} R^{\mu \nu \rho \sigma}$ and $R_{\mu \nu \rho \sigma} R^{\rho \sigma \lambda \tau} R_{\lambda \tau}^{\mu \nu}$. These singularities are of two kinds, spacelike singularity (e.g., Schwarzschild singularity) and timelike singularity. In timelike singularities, two possibilities arise. (i) There are an even number of horizons around a timelike singularity (e.g., RN black hole). In that case, an observer cannot see this singularity from outside (the singularity is covered by an event horizon). (ii) There is no horizon around a timelike singularity and the latter one may be observable from the rest of the spacetime.

To counter the occurrence of timelike singularities which are not covered by an event horizon, Penrose [1-3] proposed a Conjecture known as Cosmic Censorship Conjecture. The Cosmic Censorship Conjecture (CCC) essentially states that no physically realistic collapse, evolving from a well-posed initial data set and satisfying the dominant energy condition, results in a singularity in the causal past of null infinity.
According to Weak Cosmic Censorship, singularities have no effect on distant observers; that is, they cannot communicate with far-away observers. Till date, there are no mathematical theorems or proofs that support (or counter) this Conjecture. On the contrary, there is no mathematical reason that a naked singularity cannot exist in a solution of the field equations in GR. In literature, there are a number of examples of gravitational collapse which form a naked curvature singularity. The earliest model is the Lemaitre-Tolman-Bondi (LTB) [46] solution, a spherically symmetric inhomogeneous collapse of dust fluid that admits both naked and covered singularities. A small sample of gravitational collapse with the formation of a naked singularity is discussed in [7-16]. In this paper, we construct a nonvacuum metric that forms a naked curvature singularity. The presence of a naked singularity in a solution of Einstein's field equations may break down the causality condition and may form closed timelike curves (CTCs). The possibility that a generic naked curvature singularity gives rise to a Cosmic Time Machine has been discussed by Clarke and de Felice [17] (see also [18-20]). A Cosmic Time Machine is a spacetime which is asymptotically flat and admits closed non-spacelike curves which extend to future infinity. In literature, there are a number of vacuum and nonvacuum 
spacetimes admitting CTCs which have been constructed without naked singularity. Small samples of these are in [2133]. Recently, a vacuum spacetime with a naked curvature singularity admitting CTCs appeared in [34] which may represent a Cosmic Time Machine. One way of classifying causality violating spacetimes would be to categorize the metrics either as eternal time machines in which CTCs always exist (in this class would be $[21,22]$ ) or as time machine spacetimes in which CTCs appear after a certain instant of time (in this class would be $[25,26]$ ). However, many of the spacetimes admitting CTC suffer from one or more severe physical problems. The time machine spacetime discussed in [35-39] violated the weak energy condition (WEC) and the strong energy condition (SEC) is violated in [40-43]. The WEC states that for any physical (timelike) observer the energy density is nonnegative, which is the case for all known types of (classical) matter fields. Hawking proposed a Chronology Protection Conjecture [44] which states that the laws of physics will always prevent a spacetime from forming closed timelike curves (CTCs). But the general proof of the Chronology Protection Conjecture has not yet existed.

\section{Analysis of the Spacetime}

Consider the following cylindrically symmetric metric in $(t, r, \phi, z)$ coordinates:

$$
d s^{2}=\sinh ^{2} 2 r d r^{2}+d z^{2}+\sinh ^{2} r\left(d \phi^{2}-d t^{2}\right),
$$

where the units are chosen such that $c=1$ and $8 \pi G=1$. The coordinates are labelled as $x^{0}=t, x^{1}=r, x^{2}=\phi$, and $x^{3}=z$. The ranges of the coordinates are $0 \leq r<\infty,-\infty<z<\infty$, and $-\infty<t<\infty$ and $\phi$ is periodic coordinate: $\phi \in[0,2 \pi)$. The metric is Lorentzian with signature $(-,+,+,+)$ and the determinant of the corresponding metric tensor $g_{\mu \nu}$ is

$$
\operatorname{det} g=-\sinh ^{4} r \sinh ^{4} 2 r \text {. }
$$

The nonzero components of the Einstein tensor are

$$
\begin{aligned}
G_{t}^{t} & =G_{\phi}^{\phi}=G_{z}^{z}=-\frac{1}{4 \sinh ^{4} r}, \\
G_{r}^{r} & =\frac{1}{4 \sinh ^{4} r} .
\end{aligned}
$$

The scalar curvature invariants are given by

$$
\begin{aligned}
R_{\mu}^{\mu} & =R=\frac{1}{2 \sinh ^{4} r}, \\
R_{\mu \nu} R^{\mu \nu} & =\frac{1}{4 \sinh ^{8} r}, \\
R_{\mu \nu \rho \sigma} R^{\mu \nu \rho \sigma} & =\frac{3}{4 \sinh ^{8} r}, \\
R_{\mu \nu \rho \sigma ; \tau} R^{\mu \nu \rho \sigma ; \tau} & =\frac{4}{\sinh ^{12} r} .
\end{aligned}
$$

These curvature scalars and first-order differential invariants blow up (or diverge) at $r=0$, indicating the existence of a naked curvature singularity. Since the singularity occurs without an event horizon, the Cosmic Censorship Conjecture has no physical interest. These curvature scalars vanish rapidly at spatial infinity, that is, $r \rightarrow \infty$, indicating that the spacetime is asymptotically flat radially.

\section{Cylindrical Symmetry and Petrov Type of the Spacetime}

The spacetime represented by (1) is cylindrically symmetric as it is clear from the following. Consider the Killing vector $\eta=\partial_{\phi}$ which has the normal form

$$
\eta^{\mu}=(0,0,1,0)
$$

Its covector form is

$$
\eta_{\mu}=\sinh ^{2} r(0,0,1,0) .
$$

The vector (5) satisfies the Killing equation $\eta_{\mu ; \nu}+\eta_{\nu ; \mu}=0$. The spacetime (1) that has an axially symmetric axis is assured by the condition [45-49]

$$
\mathbf{X} \equiv\left\|\partial_{\phi}\right\|^{2}=\left|g_{\mu \nu} \eta^{\mu} \eta^{\nu}\right|=\left|g_{\phi \phi}\right|=\sinh ^{2} r \longrightarrow 0,
$$

as $r \rightarrow 0^{+}$, where we have chosen the radial coordinate $r$ such that the symmetry axis is located at $r=0$. In addition to this, the spacetime admits another spacelike Killing vector $\xi=\partial_{z}$ which, with the axial Killing vector $\eta=\partial_{\phi}$, generates a two-dimensional isometry group and they commute. Thus the isometry group is Abelian and the presented spacetime is cylindrically symmetric $[46,47]$. However, the spacetime is not regular near to the axis as it fails to satisfy the regularity condition (or elementary flatness condition) [4649], namely,

$$
\frac{\left(\nabla_{\mu} \mathbf{X}\right)\left(\nabla^{\mu} \mathbf{X}\right)}{4 \mathbf{X}} \longrightarrow 1,
$$

in the limit at the rotation axis, that is, $r \rightarrow 0$. The appearance of spacetime singularities on the axis can be considered as representing the existence of some kind of sources [49-53].

For the classification of metric (1), we construct the following set of a null tetrad $(k, l, m, \bar{m})$ and they are

$$
\begin{aligned}
k_{\mu} & =\frac{\sinh r}{\sqrt{2}}(1,0,1,0), \\
l_{\mu} & =\frac{\sinh r}{\sqrt{2}}(1,0,-1,0), \\
m_{\mu} & =\frac{1}{\sqrt{2}}(0, \sinh 2 r, 0, i), \\
\bar{m}_{\mu} & =\frac{1}{\sqrt{2}}(0, \sinh 2 r, 0,-i),
\end{aligned}
$$

where $i=\sqrt{-1}$. The set of null tetrads above are such that the metric tensor for the line element (1) can be expressed as

$$
g_{\mu \nu}=-k_{\mu} l_{\nu}-l_{\mu} k_{\nu}+m_{\mu} \bar{m}_{\nu}+\bar{m}_{\mu} m_{\nu} .
$$


The vectors (9) are null vectors and are orthogonal except for $k^{\mu} l_{\mu}=-1$ and $m_{\mu} \bar{m}^{\mu}=1$. Using this null tetrad above, we have calculated the five Weyl scalars, of which only

$$
\Psi_{2}=\frac{1}{12 \sinh ^{4} r}
$$

is nonvanishing, while $\Psi_{0}=\Psi_{1}=0=\Psi_{3}=\Psi_{4}$. The metric is clearly of type $\mathrm{D}$ in the Petrov classification scheme. The nonvanishing Weyl scalar $\Psi_{2}$ vanishes rapidly at spatial infinity along the radial direction, that is, $r \rightarrow \infty$, again indicating the asymptotic flatness.

\section{Matter Field Distribution of the Spacetime and Kinematic Parameters}

For spacetime (1), we consider the energy-momentum tensor for an anisotropic fluid given by

$$
T_{\mu \nu}=\left(\rho+p_{t}\right) U_{\mu} U_{\nu}+p_{t} g_{\mu \nu}+\left(p_{r}-p_{t}\right) \zeta_{\mu} \zeta_{\nu},
$$

where $U^{\mu}$ is the unit timelike four-velocity vector and $\zeta_{\mu}$ is the unit spacelike vector along the radial direction $r$. Here the physical parameters $\rho, p_{r}$, and $p_{t}$ are the energy density, the radial pressure, and the tangential pressure of the anisotropic fluid, respectively.

For metric (1), the timelike unit four-velocity vector $U^{\mu}$ is defined by

$$
\begin{aligned}
U^{\mu} & =\left(\frac{1}{\sinh r}, 0,0,0\right), \\
U^{\mu} U_{\mu} & =-1 .
\end{aligned}
$$

And the spacelike unit vector $\zeta_{\mu}$ along the radial direction is

$$
\begin{aligned}
\zeta_{\mu} & =(0, \sinh 2 r, 0,0), \\
\zeta_{\mu} \zeta^{\mu} & =1 .
\end{aligned}
$$

The nonzero components of the energy-momentum tensor (12) using (13) and (14) are

$$
\begin{aligned}
& T_{t}^{t}=-\rho, \\
& T_{r}^{r}=p_{r}, \\
& T_{\phi}^{\phi}=T_{z}^{z}=p_{t},
\end{aligned}
$$

and the trace of the energy-momentum tensor (12) is

$$
T_{\mu}^{\mu}=T=p_{r}+2 p_{t}-\rho
$$

Einstein's field equations (taking cosmological constant $\Lambda=$ 0 ) are given by

$$
G^{\mu \nu}=T^{\mu \nu}, \quad \mu, \nu=0,1,2,3,
$$

where $G^{\mu \nu}$ is the Einstein tensor and $T^{\mu \nu}$ is the energymomentum tensor. Thus we have from the field equations (17) using (3), (15), and (16)

$$
\begin{aligned}
\rho & =\frac{1}{4 \sinh ^{4} r}, \\
p_{r} & =\frac{1}{4 \sinh ^{4} r}, \\
p_{t} & =-\frac{1}{4 \sinh ^{4} r}, \\
T & =-R,
\end{aligned}
$$

which are diverging at $r=0$, a fact that indicates the collapse of anisotropic fluid on the symmetry axis. The matter distribution satisfies the different energy conditions [54]:

$$
\begin{aligned}
\text { WEC }: & \rho>0, \\
\text { WEC }_{t}: & \rho+p_{t}=0, \\
\text { WEC }_{r}: & \rho+p_{r}>0, \\
\text { SEC }: & \rho+p_{r}+2 p_{t}=0, \\
\text { DEC }: & \rho=\left|p_{j}\right|, \quad j=t, r .
\end{aligned}
$$

The kinematic parameters, the expansion $\Theta$, the acceleration vector $\dot{U}^{\mu}$, the shear tensor $\sigma_{\mu \nu}$, and the vorticity tensor $\omega_{\mu \nu}$ associated with the fluid four-velocity vector are defined by

$$
\begin{aligned}
\Theta & =U_{; \mu}^{\mu}, \\
a^{\mu} & =\dot{U}^{\mu}=U^{\mu ; \nu} U_{\nu}, \\
\sigma_{\mu \nu} & =U_{(\mu ; \nu)}+\dot{U}_{(\mu} U_{\nu)}-\frac{1}{3} \Theta h_{\mu \nu}, \\
\omega_{\mu \nu} & =U_{[\mu ; v]}+\dot{U}_{[\mu} U_{\nu]},
\end{aligned}
$$

where $h_{\mu \nu}=g_{\mu \nu}+U_{\mu} U_{\nu}$ is the projection tensor. For spacetime (1), these parameters have the following expressions:

$$
\begin{aligned}
& \Theta=0=\sigma_{\mu \nu}=\omega_{\mu \nu}, \\
& a_{\mu}=\operatorname{coth} r \delta_{\mu}^{r} .
\end{aligned}
$$

The magnitude of the acceleration vector $a^{2}=a^{\mu} a_{\mu}$ is $a=$ $(1 / 2) \operatorname{csch}^{2} r$.

\section{Generating a Rotating Spacetime Admitting CTCs: A Cosmic Time Machine}

One can easily generate a rotating spacetime mixing of the $d T d \psi$ term from metric (1) by the following transformations, keeping $r$ and $z$ the same:

$$
\begin{gathered}
t \longrightarrow-e^{-\psi / 2}+T e^{\psi / 2} \\
\phi \longrightarrow e^{-\psi / 2}+T e^{\psi / 2} .
\end{gathered}
$$


From metric (1), we get

$$
d s^{2}=\sinh ^{2} 2 r d r^{2}+d z^{2}-\sinh ^{2} r\left(T d \psi^{2}+2 d T d \psi\right)
$$

where $\psi$ is periodic coordinate and $-\infty<T<\infty$.

Consider an azimuthal curve $\gamma$ defined by $r=r_{0}, z=$ $z_{0}, T=T_{0}$, where $r_{0}>0, z_{0}$, and $T_{0}$ are constants. From (23), we have

$$
d s^{2}=-T \sinh ^{2} r d \psi^{2}
$$

These curves are null (or null geodesics) at $T=T_{0}=0$, spacelike throughout $T=T_{0}<0$, but become timelike for $T=T_{0}>0$, which indicates the presence of CTCs. Hence, CTCs form at a definite instant of time satisfying $T=T_{0}>0$. The above analysis is valid provided that the CTCs evolve from an initially spacelike $T=$ const hypersurface [26]. A hypersurface $T=$ const is spacelike provided $g^{T T}<0$ for $T<0$, timelike provided $g^{T T}>0$ for $T>0$, and null hypersurface provided $g^{T T}=0$ for $T=0$. From metric (23), we found that

$$
g^{T T}=\frac{T}{\sinh ^{2} r} .
$$

Thus, the hypersurface $T=$ const $=T_{0}<0$ is spacelike $(r \neq 0)$ and can be chosen as initial hypersurface over which the initial data may be specified. There is a Cauchy horizon at $T=T_{0}=0$ for any such initial hypersurface $T=T_{0}<0$. Hence, the spacetime evolves from an initial spacelike hypersurface in a causally well-behaved manner, up to a moment, that is, a null hypersurface $T=T_{0}=0$, and the formation of CTC takes place from causally well-behaved initial conditions. Assuming that the evolution beyond the Cauchy horizon proceeds in an analytic manner, we recover the region $T>0$ as well, and CTCs appear.

The formation of CTCs is thus identical to the Misner space. The Misner space is a two-dimensional locally flat metric with peculiar identifications. The metric of Misner space in $2 \mathrm{D}[55]$ is

$$
d s_{\text {Misn }}^{2}=-2 d T d X-T d X^{2},
$$

where $-\infty<T<\infty$ but the coordinate $X$ is periodic. The curves defined by $T=T_{0}>0$, where $T_{0}$ is a constant, are timelike and closed (since $X$ is periodic); thus CTCs formed. Note that the metric of Misner space (26) can be obtained from the Minkowski metric,

$$
d s_{\text {Mink }}^{2}=-d t^{2}+d x^{2},
$$

by applying a similar transformation (22).

\section{Conclusion}

In this paper, we present a cylindrically symmetric metric solution of the field equations which is regular everywhere except on the symmetry axis where it possesses a naked curvature singularity. The spacetime is of type $\mathrm{D}$ in the Petrov classification and is asymptotically flat radially and its matterenergy content is an anisotropic fluid that satisfies the different energy conditions. The nonzero kinematic parameters associated with the fluid four-velocity vector; namely, the acceleration diverges on the symmetry axis and vanishes at large radial distance. Similarly, the physical parameters, the energy density, the radial pressure, and the tangential pressure of the anisotropic fluid, behave in the same way. Finally, we generate a rotating spacetime admitting CTCs and these curves evolve from an initial spacelike hypersurface in a causally well-behaved manner. The possibility that a naked curvature singularity gives rise to a Cosmic Time Machine has been discussed by Clarke and his collaborators [17]. The presented cylindrically symmetric spacetime may represent such a Cosmic Time Machine.

\section{Disclosure}

The author's permanent address is Hindustani Kendriya Vidyalaya, Dinesh Ojha Road, Guwahati 781 005, India.

\section{Competing Interests}

The author declares that there are no competing interests regarding the publication of this paper.

\section{References}

[1] R. Penrose, "Gravitational collapse: the role of general relativity," Revista del Nuovo Cimento, vol. 1, pp. 252-276, 1969.

[2] R. Penrose, "Singularities and time-asymetry," in General Relativity: An Einstein Centenary Survey, S. W. Hawking and W. Israel, Eds., pp. 581-638, Cambridge University Press, Cambridge, UK, 1979.

[3] R. Penrose, "The question of cosmic censorship," in Black Holes and Relativistic Stars, R. M. Wald, Ed., chapter 5, Chicago University Press, Chicago, Ill, USA, 1994.

[4] G. Lemaitre, "Lunivers en expansion," Annales de la Societe Scientifique de Bruxelles A, vol. 53, p. 51, 1933, Translated by M. A. H. MacCallum, "The expanding Universe", General Relativity \& Gravitation, vol. 29, no. 5, p. 641, 1997.

[5] R. C. Tolman, "Effect of inhomogeneity on cosmological models," Proceedings of the National Academy of Sciences of the United States of America, vol. 20, no. 3, pp. 169-176, 1934.

[6] H. Bondi, "Spherically symmetric models in general relativity," Monthly Notices of the Royal Astronomical Society, vol. 107, no. 5-6, p. 410, 1947.

[7] A. Papapetrou, "Formation of a singularity and causality," in $A$ Random Walk in Relativity and Cosmology, N. Dadhich, J. K. Rao, J. V. Narlikar, and C. V. Vishveshwara, Eds., pp. 184-191, John Wiley \& Sons, New York, NY, USA, 1985.

[8] P. C. Vaidya, “'Newtonian' time in general relativity," Nature, vol. 171, pp. 260-261, 1953.

[9] D. Christodoulou, "Violation of cosmic censorship in the gravitational collapse of a dust cloud," Communications in Mathematical Physics, vol. 93, no. 2, pp. 171-195, 1984.

[10] P. S. Joshi, Global Aspects in Gravitation and Cosmology, vol. 87 of International Series of Monographs on Physics, The Clarendon Press, Oxford, UK, 1993. 
[11] P. S. Joshi, Singularities, Black Holes and Cosmic Censorship, IUCAA Publication, Pune, India, 1997.

[12] S. S. Deshingkar, P. S. Joshi, and I. H. Dwivedi, "Physical nature of the central singularity in spherical collapse," Physical Review D, vol. 59, no. 4, Article ID 044018, 1999.

[13] K. S. Govinder and M. Govender, "Gravitational collapse of null radiation and a string fluid," Physical Review D, vol. 68, no. 2, Article ID 024034, 2003.

[14] S. Barve, T. P. Singh, C. Vaz, and L. Witten, "A simple derivation of the naked singularity in spherical dust collapse," Classical and Quantum Gravity, vol. 16, no. 6, p. 1727, 1999.

[15] C. J. Clarke, "A title of cosmic censorship," Classical and Quantum Gravity, vol. 11, no. 6, pp. 1375-1386, 1994.

[16] P. S. Joshi and I. H. Dwivedi, "Initial data and the end state of spherically symmetric gravitational collapse," Classical and Quantum Gravity, vol. 16, no. 1, pp. 41-59, 1999.

[17] C. J. Clarke and F. de Felice, "Globally noncausal space-times. II. Naked singularities and curvature conditions," General Relativity and Gravitation, vol. 16, no. 2, pp. 139-148, 1984.

[18] F. de Felice, "Cosmic time machines", in Birth of the Universe and Fundamental Physics, vol. 455 of Lecture Notes in Physics, pp. 99-102, Springer, Berlin, Germany, 1995.

[19] F. de Felice, "Naked singularities, cosmic time machines and impulsive events," Nuovo Cimento B, vol. 122, p. 481, 2007.

[20] F. de Felice, "Cosmic time machines: the causality issue," EPJ Web of Conferences, vol. 58, Article ID 01001, 4 pages, 2013.

[21] K. Gödel, "An example of a new type of cosmological solutions of Einstein's field equations of gravitation," Reviews of Modern Physics, vol. 21, pp. 447-450, 1949.

[22] W. J. van Stockum, "IX.-The gravitational field of a distribution of particles rotating about an axis of symmetry," Proceedings of the Royal Society of Edinburgh, vol. 57, pp. 135-154, 1938.

[23] F. J. Tipler, "Rotating cylinders and the possibility of global causality violation," Physical Review. D. Particles and Fields. Third Series, vol. 9, pp. 2203-2206, 1974.

[24] I. Gott, "Closed timelike curves produced by pairs of moving cosmic strings: exact solutions," Physical Review Letters, vol. 66, no. 9, pp. 1126-1129, 1991.

[25] A. Ori, "Formation of closed timelike curves in a composite vacuum/dust asymptotically flat spacetime," Physical Review D. Particles, Fields, Gravitation, and Cosmology, vol. 76, no. 4, Article ID 044002, 2007.

[26] A. Ori, "A class of time-machine solutions with a compact vacuum core," Physical Review Letters, vol. 95, no. 2, Article ID 021101, 2005.

[27] W. B. Bonnor, "An exact, asymptotically flat, vacuum solution of Einstein's equations with closed timelike curves," Classical and Quantum Gravity, vol. 19, no. 23, pp. 5951-5957, 2002.

[28] W. B. Bonnor, "Closed timelike curves in classical relativity," International Journal of Modern Physics D, vol. 12, no. 09, pp. 1705-1708, 2003.

[29] W. B. Bonnor and B. R. Steadman, "Exact solutions of the Einstein-Maxwell equations with closed timelike curves," General Relativity and Gravitation, vol. 37, no. 11, pp. 1833-1844, 2005.

[30] C. W. Misner and A. H. Taub, "A singularity-free empty Universe," Soviet Physics-JETP, vol. 28, no. 1, p. 122, 1969.

[31] R. P. Kerr, "Gravitational field of a spinning mass as an example of algebraically special metrics," Physical Review Letters, vol. 11, pp. 237-238, 1963.
[32] D. Sarma, M. Patgiri, and F. U. Ahmed, "Pure radiation metric with stable closed timelike curves," General Relativity and Gravitation, vol. 46, article no. 1633, 2014.

[33] F. Ahmed, B. Bikash Hazarika, and D. Sarma, "The anti-de Sitter spacetime as a time machine," European Physical Journal Plus, vol. 131, no. 7, article no. 230, 2016.

[34] D. Sarma, F. Ahmed, and M. Patgiri, "Axially symmetric, asymptotically flat vacuum metric with a naked singularity and closed timelike curves," Advances in High Energy Physics, vol. 2016, Article ID 2546186, 4 pages, 2016.

[35] M. S. Morris, K. S. Thorne, and U. Yurtsever, "Wormholes, time machines, and the weak energy condition," Physical Review Letters, vol. 61, no. 13, pp. 1446-1449, 1988.

[36] M. S. Morris and K. S. Thorne, "Wormholes in spacetime and their use for interstellar travel: a tool for teaching general relativity," American Journal of Physics, vol. 56, no. 5, pp. 395412, 1988.

[37] M. Alcubierre, “The warp drive: hyper-fast travel within general relativity," Classical and Quantum Gravity, vol. 11, no. 5, pp. L73L77, 1994.

[38] A. E. Everett, "Warp drive and causality," Physical Review. D. Third Series, vol. 53, no. 12, pp. 7365-7368, 1996.

[39] A. E. Everett and T. A. Roman, "Superluminal subway: the Krasnikov tube," Physical Review. D, vol. 56, no. 4, pp. 21002108, 1997.

[40] Y. Soen and A. Ori, "Improved time-machine model," Physical Review D, vol. 54, no. 8, pp. 4858-4861, 1996.

[41] K. D. Olum, “Ori-Soen time machine," Physical Review D, vol. 61, no. 12, Article ID 124022, 2000.

[42] A. Ori and Y. Soen, "Causality violation and the weak energy condition," Physical Review D, vol. 49, no. 8, pp. 3990-3997, 1994.

[43] A. Ori, "Must time-machine construction violate the weak energy condition?" Physical Review Letters, vol. 71, p. 2517, 1993.

[44] S. W. Hawking, "Chronology protection conjecture," Physical Review D, vol. 46, no. 2, pp. 603-611, 1992.

[45] B. Carter, "The commutation property of a stationary, axisymmetric system," Communications in Mathematical Physics, vol. 17, pp. 233-238, 1970.

[46] M. Mars and J. M. M. Senovilla, "Axial symmetry and conformal Killing vectors," Classical and Quantum Gravity, vol. 10, no. 8, pp. 1633-1647, 1993.

[47] J. Carot, J. M. Senovilla, and R. Vera, "On the definition of cylindrical symmetry," Classical and Quantum Gravity, vol. 16, no. 9, pp. 3025-3034, 1999.

[48] A. Wang, "Critical collapse of a cylindrically symmetric scalar field in four-dimensional Einstein's theory of gravity," Physical Review D, vol. 68, no. 6, Article ID 064006, 12 pages, 2003.

[49] H. Stephani, D. Kramer, M. MacCallum, C. Hoenselaers, and E. Herlt, Exact Solutions of Einstein's Field Equations, Cambridge University Press, Cambridge, UK, 2003.

[50] W. B. Bonnor, "Physical interpretation of vacuum solutions of Einstein's equations. Part I. Time-independent solutions," General Relativity and Gravitation, vol. 24, no. 5, pp. 551-574, 1992.

[51] W. B. Bonnor, J. B. Griffiths, and M. A. MacCallum, "Physical interpretation of vacuum solutions of Einstein's equations. II. Time-dependent solutions," General Relativity and Gravitation, vol. 26, no. 7, pp. 687-729, 1994. 
[52] M. F. da Silva, L. Herrera, F. M. Paiva, and N. O. Santos, "The Levi-Civita space-time," Journal of Mathematical Physics, vol. 36, no. 7, pp. 3625-3631, 1995.

[53] S. Haggag and F. Desokey, "Perfect-fluid sources for the LeviCivita metric," Classical and Quantum Gravity, vol. 13, no. 12, p. 3221, 1996.

[54] S. W. Hawking and G. F. Ellis, The Large Scale Structure of SpaceTime, Cambridge University Press, Cambridge, UK, 1973.

[55] C. W. Misner, "Taub-NUT space as a Counter-example to almost anything," in Relativity Theory and Astrophysics I: Relativity and Cosmology, J. Ehlers, Ed., vol. 8 of Lectures in Applied Mathematics, p. 160, American Mathematical Society, 1967. 

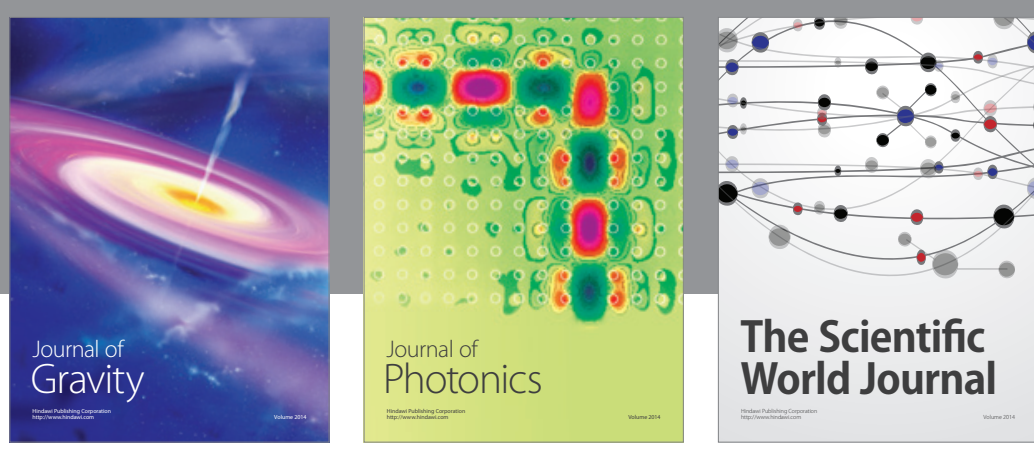

The Scientific World Journal
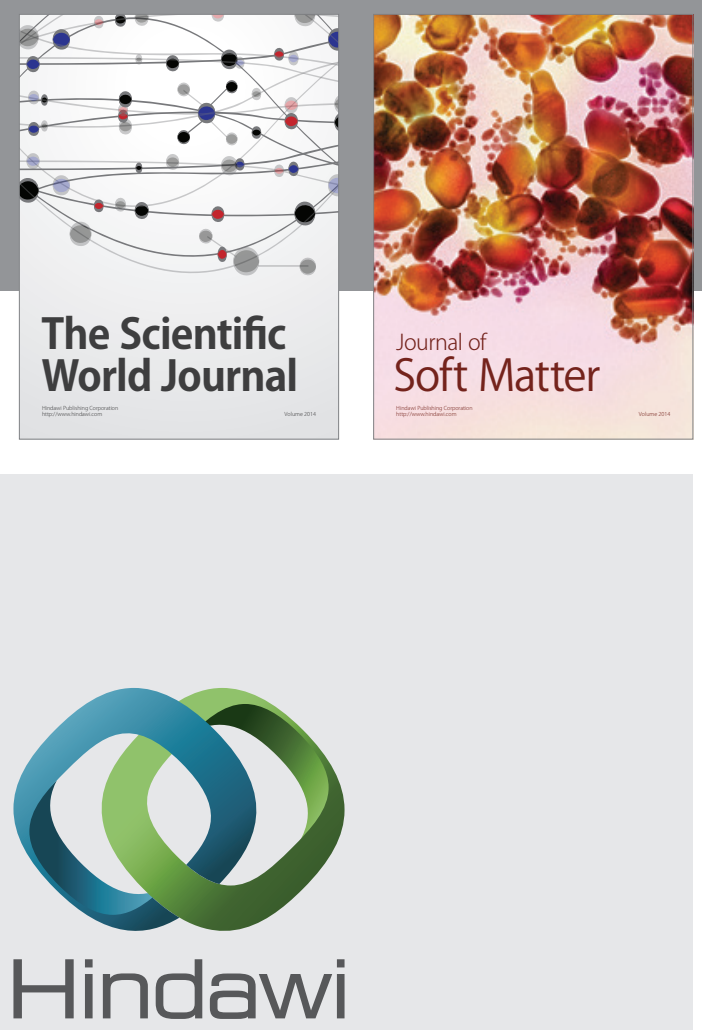

Submit your manuscripts at

https://www.hindawi.com
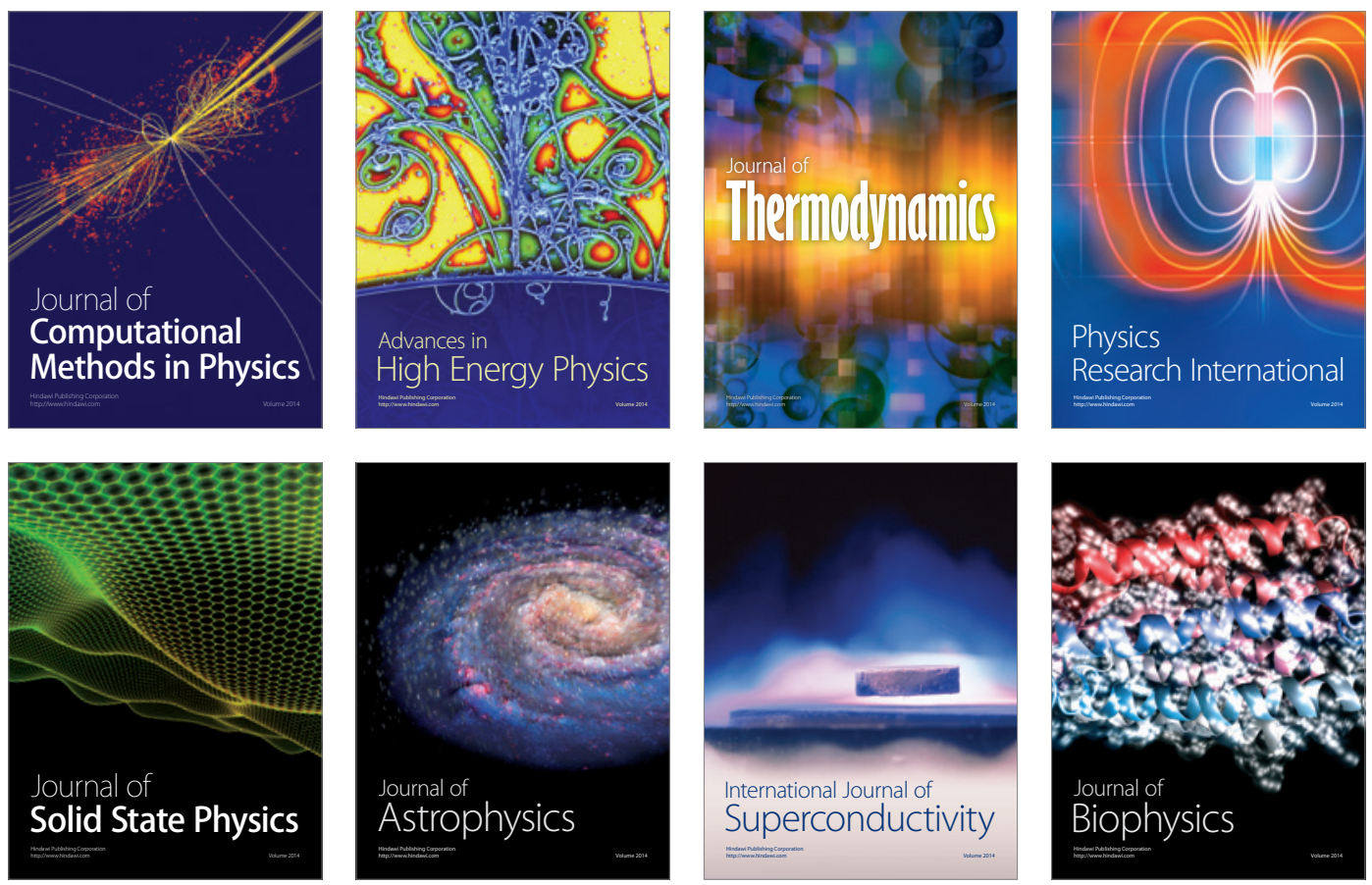
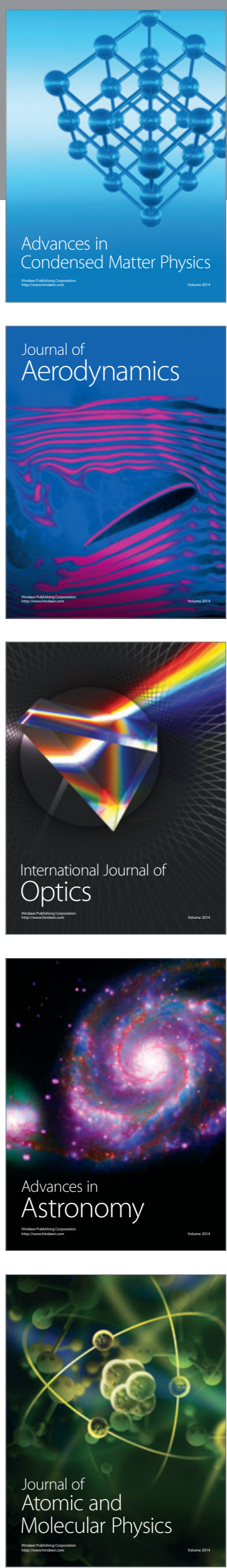